East African Medical Journal Vol. 86 No. 2 February 2009

DETERMINANTS IN HIV COUNSELLING AND TESTING IN COUPLES IN NORTH RIFT, KENYA

P. O. Ayuo, MBChB, MMed, Senior Lecturer, Department of Medicine, E. Were, MBChB, MMed, Department of Reproductive Health, School of Medicine, Moi University, P. O. Box 4606 - 30100, Eldoret, Kenya., K. Wools-Kaloustian, MD, Division of Infectious Diseases, Indiana University School of Medicine, USA., J. Baliddawa, MSc, Department of Behavioural Sciences, School of Medicine, Moi University, P. O. Box 4606 - 30100, Eldoret, Kenya, J. Sidle, MD, Division of General Internal Medicine and Geriatrics, Indiana University School of Medicine, USA and K. Fife, MD, Division of Infectious Diseases, Indiana University School of Medicine, USA

Request for reprints to: Dr. P. O. Ayuo, Department of Medicine, School of Medicine, Moi University, P. O. Box 4606 - 30100, Eldoret, Kenya

\title{
DETERMINANTS IN HIV COUNSELLING AND TESTING IN COUPLES IN NORTH RIFT KENYA
}

\author{
P. O. AYUO, E. WERE, K. WOOLS-KALOUSTIAN, J. BALIDDAWA, J. SIDLE and K. FIFE
}

\begin{abstract}
Background: Voluntary HIV counselling and testing (VCT) has been shown to be an acceptable and effective tool in the fight against HIV/AIDS. Couple HIV Counselling and Testing (CHCT) however, is a relatively new concept whose acceptance and efficacy is yet to be determined.

Objective: To describe factors that motivate couples to attend VCT as a couple.

Design: A cross sectional qualitative study.

Setting: Moi Teaching and Referral Hospital and Moi University, School of Medicine, Eldoret, Kenya

Subjects: Seventy one individuals were interviewed during KII (9) and dyad interviews (31 couples). Ten FGDs involving a total of 109 individuals were held.

Results: Cultural practices, lack of CHCT awareness, stigma and fear of results deter CHCT utilisation. Location of centre where it is unlikely to be associated with HIV testing, qualified professional staff and minimal waiting times would enhance CHCT utilisation.

Conclusions: CHCT as a tool in the fight against HIV/AIDS in this region of Kenya is feasible as the factors that would deter couples are not insurmountable.
\end{abstract}

\section{INTRODUCTION}

Voluntary counselling and testing (VCT) is the process by which a person undergoes counselling to enable him/her make an informed choice about being tested for HIV. The overall goal of VCT is to prevent the spread of HIV / AIDS as well as mitigate morbidity and mortality in people living with HIV / AIDS (PLWHA) (1). Painter (2) pointed out that VCT is associated with reduced sexual risk behaviours and lower rates of sero-conversion among HIV serodiscordant couples in reference to findings by the Voluntary HIV-l Counselling and Testing Efficacy Study Group (3). This positive effect of VCT can be explained by, among other factors, increased support for HIV sero-positive individuals by health care workers (4). VCT as a tool in the control of HIV / AIDS has also been found to be cost effective (5).
CHCT is the simultaneous counselling and testing of two individuals in a sexual relationship. When couples are tested together the "burden" of disclosure is shifted from either member of the couple to the counsellor, thus avoiding the situation of nondisclosure brought about by fear of rejection (4). Nondisclosure of a positive sero-status is common among couples in whom either membergoes for testing alone (6). This is a drawback in the fight against HIV / AIDS as it has been shown that knowledge of partner's sero-status enhances safe sexual behaviour $(3,7)$. The major challenge facing couples who test together is how to cope with discordant test results. Reactions will vary in different couples from improved relationship and safety of sex (8) to the worst possible scenario of ending the relationship (7).

In some countries like Uganda, successful acceptance of couples VCT has been demonstrated 
even in rural-based centres (8). However, advocacy for CHCT is new in Kenya (10) and therefore, widespread implementation of couples counselling is lacking throughout the nation. Consequently factors that determine CHCT utilisation in Kenya have not been fully understood. This study was therefore planned to inform a large multi-centre trial (thePIP study) whose main aim is to assess the efficacy of HSV-2 suppression with daily acyclovir on HIV transmission among HIV -discordant couples. It examined the feasibility of couple recruitment methods by answering the question: What are the factors that motivate couples to attend VCT as a couple?

\section{MATERIALS AND METHODS}

Design: This was a cross-sectional qualitative study of factors related to the recruitment of HIV-1 discordant couples for CHCT. The study employed focus groups discussions, key informant interviews and dyad interviews. The study was approved by the Institutional Research and Ethics Committee of the Moi University School of Medicine as well as the Institutional Review Board of Indiana University.

Study setting: Participants were recruited from Eldoret, a city of approximately 450,000 people located about 300 kilometres west of Nairobi, Kenya. Both Moi Teaching and Referral Hospital and Moi University School of Medicine are located in Eldoret and jointly offer voluntary counselling and testing (VCT), prevention of mother to child transmission (PMTCT) and HIV clinical services (including anti-retroviral drugs) for the community surrounding Eldoret. In addition, there are Ministry of Health run VCT services at ten other health centres within the District.

Study population: Participants were identified from VCT centres and the communities surrounding the sites. Index dyad members wereidentified at the time of visits to a participating VCT centre or clinic and requested to participate in the study. Those willing were requested to return with their partners on appointed day where upon they were asked about possible participation in a focus group discussion (FGD) and dyad interview. Interested couples were referred to a research assistant, who privately explained the purpose and procedures involved. Couples whoexpressed understanding and continued willingness to participate were scheduled for the interview. Dyads were chosen even if not sexually active at the time.
The Community leaders, health administrators and care providers for FGDs and key informant interviews (KIIs) were purposefully sought and matched by status or cause, for instance, all 8-12 memberswould be chiefs (governmentadministrators in charge of a location within a district) or chairpersons of women's groups or religious leaders. Through the same community leaders, entry was gained into various groups who were recruited for FGDs.

Data collection and analysis: Data collection teams consisted of a moderator (facilitator), an observer, and a translator chosen from among the research assistants and outreach workers. The observer was responsible for time keeping and detailed note taking and audiotaping of the discussion (FGD) or interview sessions (KII, Dyad). With the concordant and discordant couples, it was ensured that a PLWA was the moderator. It was anticipated that this arrangement would assist in establishing an atmosphere of trust and comfort for all participants. The moderator was responsible for the preparation of venues, making the necessary appointments and bookings and controlling the sessions. Research assistants were used as interpreters when necessary.

Audiotapes were transcribed in Word 2000 by an individual fluentinall languages used for theinterview. Final transcripts for analysis were in English. The transcribers were blinded to the purpose of the study. To protect participant confidentiality, no names were transcribed in the final analysis of audiotapes. The transcripts were saved and transmitted in password protected files by the transcribers to the analysts. Two experienced behavioural scientists performed the analysis. They analysed the transcripts after confirming their accuracy through a validation process comparing the audiotapes with the transcripts. Attention was given to themes, specific language and new issues that arose from the focus groups. A constant comparison methodology was used to link potential dimensions relevant to couple VCT to those suggested by theory and by prior research.

\section{RESULTS}

Seventy one individuals were interviewed during KII (9) and dyad interviews (31 couples; 10 untested, 10 concordant and 11 discordant) as shown in Table 1. Ten FGDs involving a total of 109 individuals were held as summarised in Table 2. A summary of the key findings is given together with representative quotes. 
Table 1

Type and number of respondents

\begin{tabular}{|c|c|c|}
\hline Strategy & Category of participants & No. \\
\hline \multirow[t]{12}{*}{ KII } & Director of HIV clinical & \\
\hline & programme & 1 \\
\hline & VCT Officer, Referral & \\
\hline & Hospital & 1 \\
\hline & District Officers & 2 \\
\hline & District Commissioner & 1 \\
\hline & Medical Officer of & \\
\hline & Health, Uasin Gishu District & 1 \\
\hline & Clinical Officer & \\
\hline & Mosoriot Health Centre & 1 \\
\hline & Traditional Birth Attendants & 2 \\
\hline & Total & 9 \\
\hline \multirow[t]{5}{*}{ Dyad interviews } & Untested couples & 10 \\
\hline & Concordant positive couples & 7 \\
\hline & Concordant negative couples & 3 \\
\hline & Discordant couples & 11 \\
\hline & Total & 31 couples $=62$ individuals \\
\hline
\end{tabular}

Table 2

Group composition and number of FGD (per site)

\begin{tabular}{llcc}
\hline Group composition & Membership & No. of FGDs & $\begin{array}{c}\text { No. of } \\
\text { participants }\end{array}$ \\
\hline Decision makers & District administrators & 1 & 9 \\
Community leaders & Chiefs / assistant chiefs & 2 & 18 \\
& Religious leaders & 1 & 9 \\
Community based & Women groups & 2 & 25 \\
organisations & Men groups & 2 & 25 \\
& Youth groups & 2 & 25 \\
Untested couples & & 3 & 36 \\
Discordant couples & & 1 & 12 \\
\hline Total & & 10 & 109 \\
\hline
\end{tabular}

Barriers to CHCT Utilisation:In many African societies there are clearly defined roles and modes of behaviour for men and women, and it is not usual to find men and women mingling easily to the extent that in some communities husband and wife cannot walk with each other even when going to the same place.

This cultural practice presents a significant barrier to $\mathrm{CHCT}$ in the community where this study was undertaken as captured by the quotes below: "Most of our African communities are such that ... men are not free to go with their wives to whatever service". (A community leader during KII).
" ... something we must say, among the Kalenjin (Kalenjin is the dominant tribe in the catchment population), to walk with wife ... there is nothing like that". (FGD session with community leaders)

The interviews have revealed that there is lack of awareness of CHCT. There was general consensus that the public needs to be informed about CHCT. This need was captured by the following:

"I think there should be a way of telling the public what services are available and what are the benefits of CHCT" (KII). "It is important to have the community sensitised on, first of all what is VCT and particularly 
the need for a couple to go for VCT as a couple" (KII, Health administrator.

This publiceducation may take the form of barazas (open air public meeting) or seminars during which the positives and negatives of CHCT are addressed. Some saw a major role for community leaders in this respect, thus as suggested by the following:

“... these local leaders shall think they should get the information first, once they get the information, it will be easier (for them) to pass it to the local people" (untested couple). Most interviewees alluded to stigma as a barrier by expressing that centres should be located where they are not associated with HIV testing. "Because the moment you are seen entering there (the testing centre), and the stigma associated with HIV / AIDS no body would like to go and sit in the VCT and be seen by people ... " (KII, Decision Maker).

Most people express fear of receiving a positive testresult. The fear was typified by the quote, "Because I have seen many people .., some of whom say that' if I am tested, I will know that I have it (HIV) and I will die faster" (Dyad interview; untested couple).

What would motivate couples to seekCHCT? Participants indicated that the most desirable facility would be close to those whom it provided services and be easily accessible and should not be in a place that is identifiable as an HIV testing site. Some even suggested home testing to avoid having to travel as a couple to the testing centre: "If you have a mobile unit or a way of testing at home the idea of walking to a clinic where anyone who goes there is considered to be sick or having something wrong will be avoided" (KII, decision maker). There were two different views as to the ideal location of a CHCT. The first is that CHCT centres should be located at the health centres where many services are offered and as such are less likely to be directly associated with HIV testing, and the second is that centres should be in a private/ secluded/anonymous place where attendees will not be seen. The bottom line is that nobody wishes to be seen going to a centre known to everybody as an HIV test centre. These views were embodied in the following quotes: "It should be accessible ... but not quite conspicuous to everybody" (Dyad interview, untested couple). "What will someone think when he/ she sees me queuing or entering the rooms ( $\mathrm{CHCT}$ room)" (KII).

Respondents felt that the staff manning the centres should be well trained especially in couples counselling and exhibit professionalism at all times. Ideally, they should be of the same age group as potential clients. It was felt that these characteristics in the staff would enhance client confidence and negate fears about confidentiality. On the preferred age of the counsellor the respondents said,
"Like in this FPA (a testing centre), the in charge is like my fourth born child (in age), how do you expect him to counsel me?" (FGD, CBO members).

Community leaders also concurred with this counsellor age requirement: "You cannot send a young girl like these ones (age of moderator below 30 years) to an elderly man and he agrees to say how he is" [FGD Community leaders].

Lack or breach (perceived or real) of confidentiality was identified as a major deterrent to $\mathrm{CHCT}$, as represented by the following quote: "If you go for testing and they reveal your details to other people then that is one way that will make people not to go there" (KII). Participants indicated that HIV testing should be prompt and most preferably done on the same day in order to reduce the amount of time spent at the facility. To achieve this at least one counsellor should be dedicated to serving couples. Participants expressed the general opinion that it takes a lot of effort and courage for a couple to attend CHCT together and as such the providers should also put extra effort to serve the couples in a timely manner. A key quote expressing this view was:

"... that for two members (couple) to have talked and agreed to come for a test together, it must have cost them something" (KII).

Availability of HIV/AIDS clinical services including on-going counselling and ARV provision after one tests positive and living examples of couples who tested together and are seen in the community to be doing well in health and socially were also identified as factors that would enhance CHCT utilisation.

\section{DISCUSSION}

This study has shown that CHCT concept in the control of HIV / AIDS is acceptable to the community. However, there are certain issues that must be addressed to ensure that couples attend the CHCT services as couples. The issues identified can be broadly classified into two categories; i) barriers (perceived or real) to $\mathrm{CHCT}$ and ii) factors that will attract couples to the CHCT centres.

Barriers: Most respondents alluded to stigma in the interviews both directly and indirectly. Stigma therefore is still a significant factor that will hamper the success of CHCT. Evidence for stigma can be deduced from the advocated sites of CHCT centres. Most people felt that the centres should be located in isolated non-conspicuous areas having no signs or in areas used by a lot of people such as the existing health centres. There is also evidence of stigma when respondents discuss the type or qualities of counsellors manning the sites. Thus most of the wishes of the respondents regarding site of $\mathrm{CHCT}$ 
and qualification of counsellors manning them were aimed partly at addressing stigma associated with HIV test. This is in agreement with a study by Laver (11) of rural adult Zimbabweans' preparedness for HIV testing in which fear of being seen by friends and stigmatisation were reported to be among the major factors preventing people from visiting VCT centres. Similar findings were reported by Day et al. (12) in a study of attitudes to HIV among mine workers in South Africa.

People naturally exhibit anxiety should their test for any disease turn positive. But if the disease in question has nocure and is amenable to stigmatisation such as HIV infection, then this anxiety takes a different turn and becomes fear. This is bad enough for a single person going for HIV test, but takes a different dimension for couples who turn out to be discordant. Handling discordant HIV test results is the major drawback to CHCT success. Nuwaha et al. (13) in a study in Bushenyi District in Uganda found that there are severe negative consequences to a positive HIV test result while Grinstead et al. (4) demonstrated that these negative consequences include possibility of ending relationships if a couple's result is discordant. The anticipated negative consequences fuel the fear of testing positive Day et al (12). Fear of positive result in our study is captured during interview with an untested dyad who confessed to having heard some people expressing fear that knowing their status would make them die faster. This may be the main reason why most people remain untested despite country-wide campaigns. Fortunately, it has been shown by Grinstead et al. (4) that positive health and social life events are more common than the negative ones therefore potential users of CHCT should be reassured that their fears are unwarranted.

The study area is inhabited predominantly by a community in whom cultural practices are still deeply rooted. The belief that a man should not walk with the female partner "to whatever service" is the antithesis of the CHCT principle and has been identified as posing a major threat to couples going to the testing centres as couples. Many respondents fortunately see a way out by suggesting that community leaders should be educated to be examples by ignoring this cultural belief for others to follow. In the community under study, like many in Africa, the male partner of a couple is the decision maker and in the majority of cases the sole bread earner. This means that the male partner will inevitably determine the couple's decision to attend CHCT. In Bushenyi District of Uganda, Nuwaha et al. (13) found that it takes the influence of one sexual partner for the couple to use VCT services. Therefore, in our set up, intervention targeted at the male member of a couple may positively influence utilisation of CHCT.
VCT is well known to the community but CHCT is still a new concept and many were not aware of it, let alone its advantages. Lack of awareness of CHCT is echoed by a key informant who suggested that ways and means of creating $\mathrm{CHCT}$ awareness need to be found. Awareness is the cornerstone in any endeavour that involves the community. We view $\mathrm{CHCT}$ as being parallel to VCT in terms of its introduction as a concept in HIV infection control. As such CHCT will evolve in the same way as VCT whose utilisation initially was hampered by lack of awareness $(13,14)$. Therefore for CHCT to succeed in our communities, awareness campaigns need to be mounted that vigorously focus on its advantages over VCT.

Factors that will attract couples: Suggestions by respondents on the ideal location of centres basically address two issues discussed earlier; stigma and cultural practices. Therefore the CHCT services must be as convenient as possible to the potential users. Most of our respondents suggested that for CHCT services to succeed the facilities must be close to the people utilising the services making them easily and quickly accessible. Some even proposed mobile services. These accessibility issues when tackled will also ensure that "undesirable" practice of walking with spouse is minimised by reducing the distance to the test centre. Our findings support those of Bakari et al. (15). Home testing and hidden location of centres are suggestions meant to address the issue of stigma. Similar sentiments have been found by Laver (11) and van Dyk and van Dyk (16).

People who are feeling well have no motivation to visit a health facility (17) let alone queuing for services. Fylkesnes and Siziya (18), Killewo et al. (19) and Nuwaha et al. (13) have demonstrated that utilisation of VCT for HIV is highly dependent on poor self-rated health and self-perceived risk for HIV infection. Majority of potential users of CHCT will be healthy people with no or little motivation to visit let alone spend substantial time at a health facility. Therefore, in order to augment their motivation, our respondents suggested that CHCT should not be conducted in the same place as VCT in order to enhance promptness of service and avoid referring attendees to return another day. This will also reduce the anxiety associated with waiting times for results. The realisation of this partly depends on having constant supply of testing materials and availability of adequate counselling rooms and number of couple counsellors.

Perceived quality of care has also been identified as positively influencing VCT utilisation $(4,10,13$, 14). Quality of care generally is determined by the level of training, proficiency and ethical/humane practice of the providers. Our respondents ascribe 
to this concept by suggesting that CHCT centres be manned by highly qualified staff who are "mature enough to ensure confidentiality". Preferably the staff should be of the age group of the potential clients and if possible be PL WHA to make it easy for relating during the facility visit. Similar sentiments have been documented by van Dyk and van Dyk (16). Despite all efforts over the years there is still a lot of real or perceived stigma against HIV / AIDS. This is a major barrier in our set-up for CHCT and even HIV clinical care programmes, therefore the potential user of CHCT begs for assurance on confidentiality. Thus age group and HIV sero-status of the counsellor are an attempt at addressing the fear for potential loss of confidentiality for users who turn out to be positive.

This study was constrained by time making it impossible to constitute all planned fora. Further, the respondents might have given biased views having been subjected to a lot of HIV / AIDS awareness campaigns in the past. In addition most published literature are studies on VCT and not CHCT hence comparisons are limited.

In conclussion, this study has demonstrated that CHCT as an option in the fight against HIV / AIDS in Eldoret and its environments is feasible. Health education messages targeted at addressing barriers to CHCT utilisation should be a priority. We concur with most of our respondents that the community leaders must be in the forefront in this endeavour.

Practice implications: HIV preventive services should integrate $\mathrm{CHCT}$ as a major component and the centres should be organised and/ or sited in such a way or place to make them couples friendly to address the perceived barriers to $\mathrm{CHCT}$ utilisation.

\section{ACKNOWLEDGEMENTS}

To the Bill and Melinda Gates Foundation for funding this study through the Partners in Prevention (PIP) study and Dr. Connie Celum and the University of Washington coordinating centre for the PIP study. None of the authors had any conflict of interest.

\section{REFERENCES}

1. National AIDS and STD Control Programme (NASCOP) 2003 - 2007. Kenya National Strategy for VCT Scale-Up. Ministry of Health 2004.

2. Painter, T. M. Voluntary counselling and testing for couples: a high leverage intervention for HIV / AIDS prevention in sub-Saharan Africa. Soc. Sci.Med.2001; 53: 1397-1411.

3. The Voluntary HIV-1 Counselling and Testing Efficacy Study Group. Efficacy of voluntary HIV -1 counselling and testing in individuals and couples in Kenya, Tanzania, and Trinidad: a randomised trial. Lancet. 2000; 356: 103-112.
4. Grinstead, O. A., Gregorich, S. E., Choi, K. and Coates, T. the Voluntary HIV-1 counselling and testing efficacy group. Positive and negative life events after counselling and testing: the Voluntary HIV -1 counselling and testing efficacy study. AIDS. 2001; 15: 1045-1052.

5. Sweat, M., Gregorich, S., Sangiwa, G. et al. Cost effectiveness of voluntary HIV -1 counselling and testing in reducing sexual transmission of HI V-I in Kenya and Tanzania. Lancet. 2000; 356: 86-87.

6. Wenger, N. S., Kusseling, F. S., Beck, K. and Shapiro, M. F. Sexual behaviour of individuals infected with human immunodeficiency virus. The need for intervention. Arch. Intern. Med. 1994; 154: 1849-1954.

7. Roth, D. L., Stewart, K. E., Clay, O. J., et al. Sexual practices of HIV discordant and concordant couples in Rwanda: effects of a testing and counselling programme for men. Int. J. STD AIDS. 2001; 12: 181-188.

8. Allen, S., Meinzen-Derr, J., Kautzman, M., et al.Sexual behaviour of discordant couples after HIV counselling and testing. AIDS. 2003; 17: 733-740.

9. Matovu, J. K., Kigozi, G., Nalugoda, F., WabwireMangen, F. and Gray, R. H. The Rakai Project counselling programme experience. Trop. Med. E Int. Health. 2002; 7: 1064-1067.

10. National AIDS and STD Control Programme (NASCOP). National Guidelines for Voluntary Counselling and Testing: NASCOP. Kenya. Ministry of Health 2001.

11. Laver, S. M. Voluntary testing and counselling for HIV. "Are adults in rural communities ready to test?" A descriptive survey. Cent. Afr. J. Med. 2001; 47: 92 -97.

12. Day, J. H., Miyamura, K., Grant, A. D., et al. Attitudes to HIV voluntary counselling and testing among mine workers in South Africa: will availability of antiretroviral therapy encourage testing? AIDS Care. 2003; 15: 665-672.

13. Nuwaha, F., Kabatesi, D., Muganwa, M. and Whalen, C. C. Factors influencing acceptability of voluntary counselling and testing for HIV in Bushenyi District of Uganda. East Afr. Med. J. 2002: 79: 626-632.

14. Mckenna, S. L., Muyinda, G. K., Roth, D., et al. Rapid HIV testing and counselling for voluntary testing centres in Africa. AIDS. 1997; Suppl 1: S103 - S110.

15. Bakari, J. P., Mckenna, S., Myrick, A., et al. Rapid voluntary testing and counselling for HIV. Acceptability and feasibility in Zambia antenatal care clinics. Ann. NY. Acad. Sci. 2000; 918: 64-76.

16. van Dyk, A. G. and van Dyk, P. J. To know or not to know: Service-related barriers to voluntary HIV counselling and testing (VCT). South Africa Curationis. 2003; 26: 4- 10 .

17. Ewart, C. K. Social action theory for a public health psychology. Amer. Psychol. 1991; 46: 931-946.

18. Fylkesnes, K. and Siziya, S. A randomized trial on acceptability of voluntary counselling and testing. Trop. Med. Intern. Health. 2004, 9: 566 - 571.

19. Killewo, J. Z. J., Kwesigabo, G., Comoro, C., et al. Acceptability of voluntary HIV testing with counselling in a rural village in Kagera, Tanzania. AIDS Care. 1998; 10: 431-439. 